\title{
Exceptional Response to Nanoparticle Albumin-Bound Paclitaxel and Gemcitabine in a Patient with a Refractory Adenocarcinoma of the Ampulla of Vater
}

\author{
Markus Kapp ${ }^{a} \quad$ Aleksander Kosmala $^{\mathrm{b}}$ Stefan Kircher ${ }^{\mathrm{c}}$ Verena Luber ${ }^{\mathrm{a}}$ \\ Volker Kunzmann ${ }^{\text {a }}$ \\ ${ }^{a}$ Division of Medical Oncology, Department of Internal Medicine II, and ${ }^{b}$ Institute of \\ Radiology, University Hospital Würzburg, and 'Institute of Pathology, University of \\ Würzburg and Comprehensive Cancer Center Mainfranken, Würzburg, Germany
}

\section{Key Words}

Adenocarcinoma of the ampulla of Vater - Intestinal-type adenocarcinoma - Pancreatobiliary type · FOLFOX · FOLFIRI · Gemcitabine · Nanoparticle albumin-bound paclitaxel

\begin{abstract}
Ampullary carcinoma is a rare tumor and evidence on the treatment of recurrent metastatic disease is scarce. We report the case of a 60-year-old patient with an R0-resected nodepositive adenocarcinoma of the papilla of Vater of an initially diagnosed intestinal subtype who developed pulmonary metastases 2 months after adjuvant gemcitabine chemotherapy and, subsequently, liver metastases. Palliative combination chemotherapy with standard regimens for intestinal-type adenocarcinoma (FOLFOX and FOLFIRI) failed. However, subsequent combination chemotherapy with nanoparticle albumin-bound paclitaxel and gemcitabine, a regimen with proven efficacy in metastatic adenocarcinoma of the pancreas, resulted in a durable, very good partial remission. Treatment was manageable and well tolerated. Primary tumor and metastatic tissue were reassessed by immunohistochemistry and had to be reclassified to a mixed phenotype containing predominant elements of the pancreatobiliary subtype. Our case suggests that combination chemotherapy with nanoparticle albumin-bound paclitaxel and gemcitabine could represent a promising option for the treatment of this rare
\end{abstract}

\section{KARGER}

Prof. Dr. Volker Kunzmann

Department of Internal Medicine II

Zentrum Innere Medizin (ZIM)

Oberdürrbacherstrasse 6, DE-97080 Würzburg (Germany)

E-Mail kunzmann_v@ukw.de 
Kapp et al.: Exceptional Response to Nanoparticle Albumin-Bound Paclitaxel and

disease and warrants further investigation within controlled clinical trials. Moreover, thorough characterization of ampullary carcinomas by histomorphology and additional immunohistochemistry should become mandatory in order to start a chemotherapeutic regimen tailored for the definitive subtype.

(C) 2016 The Author(s)

Published by S. Karger AG, Basel

\section{Introduction}

Malignancies arising within the ampullary region distal to the bifurcation of the distal common bile duct and the pancreatic duct are referred to as ampullary carcinomas or carcinomas of the ampulla of Vater. They are one subentity of periampullary carcinomas comprising ampullary carcinomas, carcinomas of the pancreas, the distal bile duct and the periampullary duodenum. Ampullary carcinomas are rare tumors and represent $0.2 \%$ of all gastrointestinal malignancies $[1,2]$. They account for only $6 \%$ of lesions that arise in the periampullary region but for $20 \%$ of all tumor-related obstructions of the common bile duct [3].

They can be further subdivided by their histological characteristics. A study of 170 ampullary carcinomas showed that the most common histological subtype was intestinal (47\%), followed by pancreatobiliary (24\%), poorly differentiated adenocarcinomas (13\%), intestinal-mucinous (8\%), and invasive papillary (5\%) [4]. Histological subtypes and immunohistochemical staining patterns do have prognostic significance. Tumors with an intestinal differentiation seem to be associated with a more favorable survival, while the survival rate of patients with tumors of the pancreatobiliary type seems to be comparable to that of patients with pancreatic cancer [5]. A retrospective cohort study in 208 patients with ampullary adenocarcinoma reported that by combination of histomolecular phenotype and lymph node status, three biological subtypes can be discriminated. Patients with a node-negative and a nonpancreatobiliary histomolecular phenotype tumor had an excellent prognosis with a 5 -year survival rate of $88 \%$, and those with a node-positive, pancreatobiliary phenotype a poor prognosis with a 5 -year survival rate of $20 \%$. The remaining patients (node-positive, nonpancreatobiliary or node-negative, pancreatobiliary phenotype) had an intermediate prognosis and a 5-year survival rate of $47 \%$ [6].

Complete resection of the tumor with negative margins (R0 resection) is a prerequisite for cure. The Whipple procedure is considered the standard surgery for ampullary cancer. Outcomes from pancreaticoduodenectomy for ampullary cancer have improved significantly with $\mathrm{R} 0$ resection rates of up to $90 \%$ and perioperative mortality rates of less than $5 \%$ in specialized centers. Long-term survival has shown to be even possible in patients with lymph node metastases or invasion beyond the duodenal wall $[7,8]$.

There is no consensus regarding the choice of adjuvant chemotherapy regimens due to the rarity of periampullary carcinomas. One option is to treat these patients according to the treatment algorithm for patients with R0-resected tumors of the pancreatic head. While chemoradiotherapy is the preferred choice in the US, European guidelines consider adjuvant chemotherapy with either gemcitabine or 5 -fluorouracil (FU) and folinic acid as the standard of care for pancreatic cancer [9]. There is one randomized trial with 428 patients with resected periampullary adenocarcinoma that compared adjuvant chemotherapy (5-FU or gemcitabine) to observation. While adjuvant chemotherapy did not result in a significantly longer survival in the primary analysis, it was associated with a significant survival benefit in multivariable analysis adjusting for prognostic variables [10].

Reported tumor recurrence rates after radical resection of ampullary cancer range from 28 to $44 \%$ [1]. There is no standard chemotherapy regimen for metastatic ampullary carci- 
Kapp et al.: Exceptional Response to Nanoparticle Albumin-Bound Paclitaxel and Gemcitabine in a Patient with a Refractory Adenocarcinoma of the Ampulla of Vater

nomas. Considering the results of the two randomized UK trials in patients with locally advanced or metastatic biliary tract cancer trials, a combination of gemcitabine plus cisplatin might be a reasonable option for some patients [11]. The choice of chemotherapy can also be guided by the histological differentiation of the tumor. Histology of an intestinal subtype can serve as the rationale for choosing chemotherapy regimens for intestinal-type adenocarcinomas such as metastatic colorectal cancer. For tumors of a pancreatobiliary subtype, regimens used in metastatic pancreatic cancer such as single-agent gemcitabine and the newer combinations FOLFIRINOX or nanoparticle albumin-bound paclitaxel plus gemcitabine could represent more appropriate options. Both combination regimens demonstrated a significant survival benefit over gemcitabine monotherapy in metastatic pancreatic carcinoma [12-14].

We report the case of a 60-year-old male with a carcinoma of the papilla of Vater of an initially diagnosed intestinal subtype who relapsed after R0 resection and adjuvant gemcitabine chemotherapy and achieved a durable, very good partial remission with nanoparticle albumin-bound paclitaxel and gemcitabine combination chemotherapy after failure of FOLFOX and FOLFIRI.

\section{Case Report}

In June 2013, a 60-year-old male patient was admitted to our center. He presented with jaundice. Apart from that, he was asymptomatic and in an excellent performance status. There was no family history of cancer. Endosonography detected a suspicious mass of the ampulla of Vater of $10 \mathrm{~mm}$. No further tumor localizations could be found in the computed tomography (CT) of the thorax and the abdomen. Laboratory findings were typical of cholestasis with an elevated concentration of total serum bilirubin to $16.6 \mathrm{mg} / \mathrm{dl}$. The level of the tumor marker CA 19-9 was increased to 2,702 U/ml.

The patient had a pylorus-preserving partial duodenopancreatectomy with simultaneous cholecystectomy, end-to-side pancreaticojejunostomy and end-to-side hepaticojejunostomy.

Pathological examination revealed an adenocarcinoma of the ampulla of Vater infiltrating the duodenum and the pancreas with lymphangiosis carcinomatosa, perineural invasion and 11/26 tumor-infiltrated lymph nodes [pT4pN1 (11/26) G3 L1 V1 Pn1 R0]. Conventional histomorphological analysis classified the tumor to be of the intestinal type (fig. 1a).

From August 2013 to February 2014, the patient received 6 months of adjuvant chemotherapy with gemcitabine. At the first follow-up visit 2 months after completion of adjuvant therapy, the level of the tumor marker CA 19-9 was slightly increased (fig. 2). A positron emission tomography-CT showed a number of small fluorodeoxyglucose-negative nodules in the lower lobe of the right lung. To confirm the metastastic situation, a diagnostic wedge resection was performed after marking one of the suspicious nodules with methylene blue and a thin wire. Histopathological examination showed a more differentiated adenocarcinoma. Overall, the histological characteristics of the metastasis matched those of the primary tumor sample (fig. 1b).

The case was rediscussed in the local multidisciplinary tumor board. Since initial conventional histopathological examinations of the primary tumor suggested an intestinal type differentiation, it was recommended to start a palliative chemotherapy protocol for intestinal type adenocarcinoma, i.e. modified FOLFOX-6 (oxaliplatin at a dose of $85 \mathrm{mg} / \mathrm{m}^{2}$, leucovorin at a dose of $400 \mathrm{mg} / \mathrm{m}^{2}$, bolus of $5-\mathrm{FU}$ at a dose of $400 \mathrm{mg} / \mathrm{m}^{2}$ followed by continuous infusion of 5-FU over $46 \mathrm{~h}$ at a dose of $2,400 \mathrm{mg} / \mathrm{m}^{2}$, every 2 weeks). No antibody against EGFR was added due to a detected uncommon KRAS exon 3 mutation. 
Kapp et al.: Exceptional Response to Nanoparticle Albumin-Bound Paclitaxel and

Restaging was performed after 3 months ( 6 applications) of FOLFOX. The level of CA 19-9 had increased slightly (fig. 2). Otherwise, there were no abnormal laboratory findings of clinical relevance. The CT scan revealed a progression of the pulmonary lesions. The patient did not suffer from tumor-associated symptoms. However, he had developed a peripheral polyneuropathy of grade 1-2 according to common toxicity criteria (CTC) under oxaliplatin-based chemotherapy.

Thus, chemotherapy was switched to the FOLFIRI regimen (irinotecan at a dose of 180 $\mathrm{mg} / \mathrm{m}^{2}$, leucovorin at a dose of $400 \mathrm{mg} / \mathrm{m}^{2}$, bolus $5-\mathrm{FU}$ at a dose of $400 \mathrm{mg} / \mathrm{m}^{2}$ followed by continuous infusion of 5-FU over $46 \mathrm{~h}$ at a dose of $2,400 \mathrm{mg} / \mathrm{m}^{2}$, every 2 weeks). Chemotherapy was tolerated without any relevant side effects. Polyneuropathy did not progress but remained stable at CTC grade 1-2. Unfortunately, restaging after 3 months (6 applications) of FOLFIRI documented a further disease progression. The CT scan showed an increase in the preexisting pulmonary metastatic spread and new liver metastases (fig. 3). The CA 19-9 level had risen to $2,992 \mathrm{U} / \mathrm{ml}$ (fig. 2).

The patient was still in an excellent clinical condition and highly motivated to continue with chemotherapy. We decided to offer him a combination chemotherapy with nanoparticle albumin-bound paclitaxel and gemcitabine which is approved for treatment of metastastic pancreatic cancer. In the first cycle, both, nanoparticle albumin-bound paclitaxel at a dose of $125 \mathrm{mg} / \mathrm{m}^{2}$ and gemcitabine at a dose of $1,000 \mathrm{mg} / \mathrm{m}^{2}$, were given on days $1,8,15,22,29$, 36 , and 43 . From the second cycle that started on day 50 , both drugs were given on days 1,8 , and 15 and repeated on day 29. Overall, chemotherapy was well tolerated. In cycle 3, the patient developed grade 3 neutropenia and grade 1 fatigue. This was managed by dose reductions as recommended in the prescribing information. Besides, the patient developed a thrombosis of the left axillary vein where the port tube had been placed. This required therapeutic anticoagulation with low-molecular-weight heparin (tinzaparin $175 \mathrm{IU} / \mathrm{kg}$ ).

After 6 months, treatment had resulted in a stable partial remission as documented by the regression of pulmonary metastases and liver metastases (fig. 3), and normalization of the CA 19-9 level (fig. 2). Subsequently, chemotherapy was deescalated to nanoparticle albumin-bound paclitaxel $100 \mathrm{mg} / \mathrm{m}^{2}$ and gemcitabine $800 \mathrm{mg} / \mathrm{m}^{2}$ on days 1 and 15, keeping repetition of the cycle on day 29. Doses were reduced to manage hematologic toxicity. Chemotherapy administration on day 8 was omitted to allow the patient more flexibility in his private life.

Given the extraordinary response to a typical therapy for metastatic pancreatic ductal adenocarcinoma, we retrospectively performed further immunohistochemical analyses of the primary tumor samples (fig. 1c, d) and the resected pulmonary metastatic lesion (not shown). Despite the initial histomorphological classification of an intestinal type, the immunohistochemical staining of the primary tumor sample showed an expression of CK7 in most parts of the tumor specimen but no staining for CK20 or CDX2. Only a small section of the sample depicted an expression of CK20 and CDX2 but negativity for CK7. In the pulmonary metastasis, the staining pattern was almost completely CK7-positive and CK20- and CDX2-negative. Therefore, the initial histomorphological diagnosis of a solely intestinal type (based on histomorphological classification) had to be revised to a mixed phenotype (pancreatobiliary predominant) in the primary tumor, while the more differentiated pulmonary metastatic lesion represented a spread of the pancreatobiliary compound only.

As of today, the patient has been treated with the modified schedule for a total of 9 months. He is still in a durable partial remission and tolerates chemotherapy without any relevant clinical problems while maintaining an excellent quality of life. In particular, the peripheral neuropathy that the patient developed under oxaliplatin has not worsened but improved from grade 1-2 to grade 1 . We plan to continue treating the patient with this schedule of nanoparticle albumin-bound paclitaxel and gemcitabine until progression. 
Kapp et al.: Exceptional Response to Nanoparticle Albumin-Bound Paclitaxel and Gemcitabine in a Patient with a Refractory Adenocarcinoma of the Ampulla of Vater

\section{Discussion}

Lymph node involvement, depth of infiltration and lymphovascular invasion but also the histological tumor type, i.e. intestinal versus pancreatobiliary subtype, are regarded as prognostic factors for ampullary cancer [1]. Based on histomorphologic/immunohistochemical features and nodal status, different prognostic subtypes of the disease have been proposed [6]. With an ampullary carcinoma of intestinal differentiation in the initial histomorphological examination and 11 of 26 involved lymph nodes our patient exhibited a node-positive, nonpancreatobiliary phenotype with an intermediate prognosis and a projected 5-year survival rate of about 50\%. A high preoperative CA 19-9 level also seems to be an independent risk factor [1]. We chose adjuvant chemotherapy with single-agent gemcitabine for our patient, which is in accordance with common clinical practice. However, this general treatment strategy may have to be revisited, in particular for ampullary tumors of the intestinal subtype. A recent retrospective study underlined that ampullary cancer cannot be seen as one separate tumor entity and showed that prognosis and response to adjuvant chemotherapy with gemcitabine essentially depends on the definite subtype [15]. In the study, gemcitabine tended to be associated with decreased overall survival in patients with a tumor of the intestinal type but with a significantly improved survival in those with a tumor of the pancreatobiliary subtype. The authors concluded that especially ampullary tumors of the pancreatobiliary type should be treated like pancreatic ductal adenocarcinoma as their tumor biology and clinical course was similar [15]. Thus, following this treatment paradigm, our patient received the appropriate adjuvant chemotherapy given the retrospectively revised histological classification.

Nevertheless, he developed a histologically confirmed early relapse after gemcitabine which was treated with standard chemotherapy regimens from the setting of colorectal carcinoma, namely FOLFOX and FOLFIRI. Our decision was based on the classification of his ampullary carcinoma as an intestinal type by conventional histomorphological diagnostics. However, treatment with both regimens did not result in the control but in progression of the disease.

We wanted to offer the patient an alternative effective chemotherapy option. But there is limited evidence regarding palliative chemotherapy of ampullary carcinomas. Given their possible origin from intestinal or pancreatobiliary tissue we decided to give a standard regimen for metastatic pancreatic cancer despite the initial histopathological diagnosis of a tumor of the intestinal type. The combination of nanoparticle albumin-bound paclitaxel and gemcitabine has become a standard regimen for the treatment of metastatic pancreatic adenocarcinoma [9]. In the pivotal MPACT trial, first-line combination chemotherapy with nanoparticle albumin-bound paclitaxel plus gemcitabine increased median overall survival to 8.7 months as compared to 6.6 months with gemcitabine alone [hazard ratio (HR) $0.72 ; \mathrm{p}<$ 0.001 ], progression-free survival to 5.5 versus 3.7 months (HR $0.69, \mathrm{p}<0.001$ ), and overall response rate to 23 versus $7 \%(\mathrm{p}<0.001)$, respectively $[13,14]$. These data were the basis for the approval of the combination in the US and EU for the first-line treatment of patients with metastatic pancreatic cancer.

Hematotoxicity and peripheral polyneuropathy are common adverse events associated with this combination. Hematotoxicity can be managed by adhering to the recommended dose adjustments according to neutrophil and thrombocyte counts. There are also clear guidelines for dose adjustments in the event of peripheral polyneuropathy [16]. According to our clinical experience, it is a manageable adverse event, which, in the majority of cases, improves after dose reduction or pausing of nanoparticle albumin-bound paclitaxel. In the 
Kapp et al.: Exceptional Response to Nanoparticle Albumin-Bound Paclitaxel and

MPACT trial, improvement of peripheral polyneuropathy to grade 1 or less occurred within a median of 29 days [14].

The combination therapy with nanoparticle albumin-bound paclitaxel/gemcitabine led to a very good partial response in our patient. Due to the intestinal subtype histology we had not really expected a response of such a magnitude. This prompted us to reassess the primary histomorphological diagnosis by additional immunohistochemical stainings. Staining with CDX2 has been shown to be of high sensitivity and specificity for the intestinal subtype and to be an independent prognostic marker for longer survival [17]. Based on the staining pattern, we revised the initial diagnosis to an ampullary carcinoma of a mixed phenotype containing predominant elements from the pancreatobiliary subtype. This diagnosis might provide the biological rationale for the success of the chemotherapy regimen with proven efficacy in metastatic pancreatic cancer.

Interestingly, the peripheral polyneuropathy which the patient had developed under oxaliplatin improved while he was treated with nanoparticle albumin-bound paclitaxel, which can be interpreted as clinical evidence for the distinct pathomechanisms of oxaliplatin and nanoparticle albumin-bound paclitaxel-induced polyneuropathy. It shows that a preexisting polyneuropathy of grade 1 or 2 acquired under oxaliplatin should not be considered a contraindication for chemotherapy with nanoparticle albumin-bound paclitaxel.

In conclusion, chemotherapy with nanoparticle albumin-bound paclitaxel plus gemcitabine was an effective and well-tolerated palliative treatment in a patient with metastatic adenocarcinoma of the ampulla of Vater. It is worthwhile to reassess the histopathological diagnosis to clarify the reasons for an ineffective treatment strategy. However, a thorough characterization of ampullary carcinomas by histomorphology and additional immunohistochemistry should become mandatory in order to start a chemotherapeutic regimen tailored for the definitive subtype in the adjuvant and in the recurrent situation. Moreover, defining the subtype of an ampullary carcinoma can provide important prognostic information such as the estimated risk of relapse of an individual patient. Clinical trials are warranted that evaluate personalized chemotherapy concepts in ampullary carcinoma prospectively.

\section{Statement of Ethics}

This study was conducted in accordance with the Declaration of Helsinki.

\section{Disclosure Statement}

The authors received editorial support in the preparation of this paper from Dr. Susanne Hell, funded by Celgene GmbH. They are fully responsible for the entire content and all editorial decisions relating to this paper.

\section{References}

1 Klein F, Jacob D, Bahra M, et al: Prognostic factors for long-term survival in patients with ampullary carcinoma: the results of a 15-year observation period after pancreaticoduodenectomy. HPB Surg 2014;2014:970234.

-2 Albores-Saavedra J, Schwartz AM, Batich K, Henson D: Cancers of the ampulla of vater: demographics, morphology, and survival based on 5,625 cases from the SEER program. J Surg Oncol 2009;100:598-605.

-3 Palazzo L: Staging of ampullary carcinoma by endoscopic ultrasonography. Endoscopy 1998;30(suppl 1):A128-A131. 


\section{Case Reports in Oncology}

\begin{tabular}{l|l}
\hline \multicolumn{2}{l}{ Case Rep Oncol 2016;9:15-24 } \\
\hline DOI: 10.1159/000443304 & $\begin{array}{l}\text { C 2016 The Author(s). Published by S. Karger AG, Basel } \\
\text { www.karger.com/cro }\end{array}$ \\
\hline
\end{tabular}

Kapp et al.: Exceptional Response to Nanoparticle Albumin-Bound Paclitaxel and Gemcitabine in a Patient with a Refractory Adenocarcinoma of the Ampulla of Vater

4 Ruemmele P, Dietmaier W, Terracciano L, et al: Histopathologic features and microsatellite instability of cancers of the papilla of vater and their precursor lesions. Am J Surg Pathol 2009;33:691-704.

5 Westgaard A, Pomianowska E, Clausen OP, Gladhaug IP: Intestinal-type and pancreatobiliary-type adenocarcinomas: how does ampullary carcinoma differ from other periampullary malignancies? Ann Surg Oncol 2013;20:430-439.

6 Chang DK, Jamieson NB, Johns AL, et al: Histomolecular phenotypes and outcome in adenocarcinoma of the ampulla of vater. J Clin Oncol 2013;31:1348-1356.

7 Yeo CJ, Sohn TA, Cameron JL, Hruban RH, Lillemoe KD, Pitt HA: Periampullary adenocarcinoma: analysis of 5-year survivors. Ann Surg 1998;227:821-831.

8 Allema JH, Reinders ME, van Gulik TM, van Leeuwen DJ, Verbeek PC, de Wit LT, Gouma DJ: Results of pancreaticoduodenectomy for ampullary carcinoma and analysis of prognostic factors for survival. Surgery 1995;117:247-253.

19 Ducreux M, Cuhna AS, Caramella C, et al: Cancer of the pancreas: ESMO Clinical Practice Guidelines for diagnosis, treatment and follow-up. Ann Oncol 2015;26(suppl 5):v56-v68.

10 Neoptolemos JP, Moore MJ, Cox TF, et al: Effect of adjuvant chemotherapy with fluorouracil plus folinic acid or gemcitabine vs observation on survival in patients with resected periampullary adenocarcinoma: the ESPAC-3 periampullary cancer randomized trial. JAMA 2012;308:147-156.

11 Valle JW, Furuse J, Jitlal M, et al: Cisplatin and gemcitabine for advanced biliary tract cancer: a meta-analysis of two randomised trials. Ann Oncol 2014;25:391-398.

12 Conroy T, Desseigne F, Ychou M, et al: FOLFIRINOX versus gemcitabine for metastatic pancreatic cancer. N Engl J Med 2011;364:1817-1825.

13 Von Hoff DD, Ervin T, Arena F, et al: Increased survival in pancreatic cancer with nab-paclitaxel plus gemcitabine. N Engl J Med 2013;369:1691-1703.

14 Goldstein D, El-Maraghi RH, Hammel P, et al: nab-Paclitaxel plus gemcitabine for metastatic pancreatic cancer: long-term survival from a phase III trial. J Natl Cancer Inst 2015;107:pii:dju413.

15 Schiergens TS, Reu S, Neumann J, et al: Histomorphologic and molecular phenotypes predict gemcitabine response and overall survival in adenocarcinoma of the ampulla of Vater. Surgery 2015;158:151-161.

16 Summary of product characteristics of ABRAXANE. http://www.ema.europa.eu/docs/en_GB/document_library/EPAR__Product_Information/human/000778/WC500020435.pdf (accessed October 12, 2015).

-17 Kumari N, Prabha K, Singh RK, Baitha DK, Krishnani N: Intestinal and pancreatobiliary differentiation in periampullary carcinoma: the role of immunohistochemistry. Hum Pathol 2013;44:2213-2219. 


\section{Case Reports in Oncology}

\begin{tabular}{l|l}
\hline Case Rep Oncol 2016;9:15-24 \\
\hline DOI: 10.1159/000443304 & $\begin{array}{l}\text { (c) 2016 The Author(s). Published by S. Karger AG, Basel } \\
\text { www.karger.com/cro }\end{array}$ \\
\hline
\end{tabular}

Kapp et al.: Exceptional Response to Nanoparticle Albumin-Bound Paclitaxel and Gemcitabine in a Patient with a Refractory Adenocarcinoma of the Ampulla of Vater

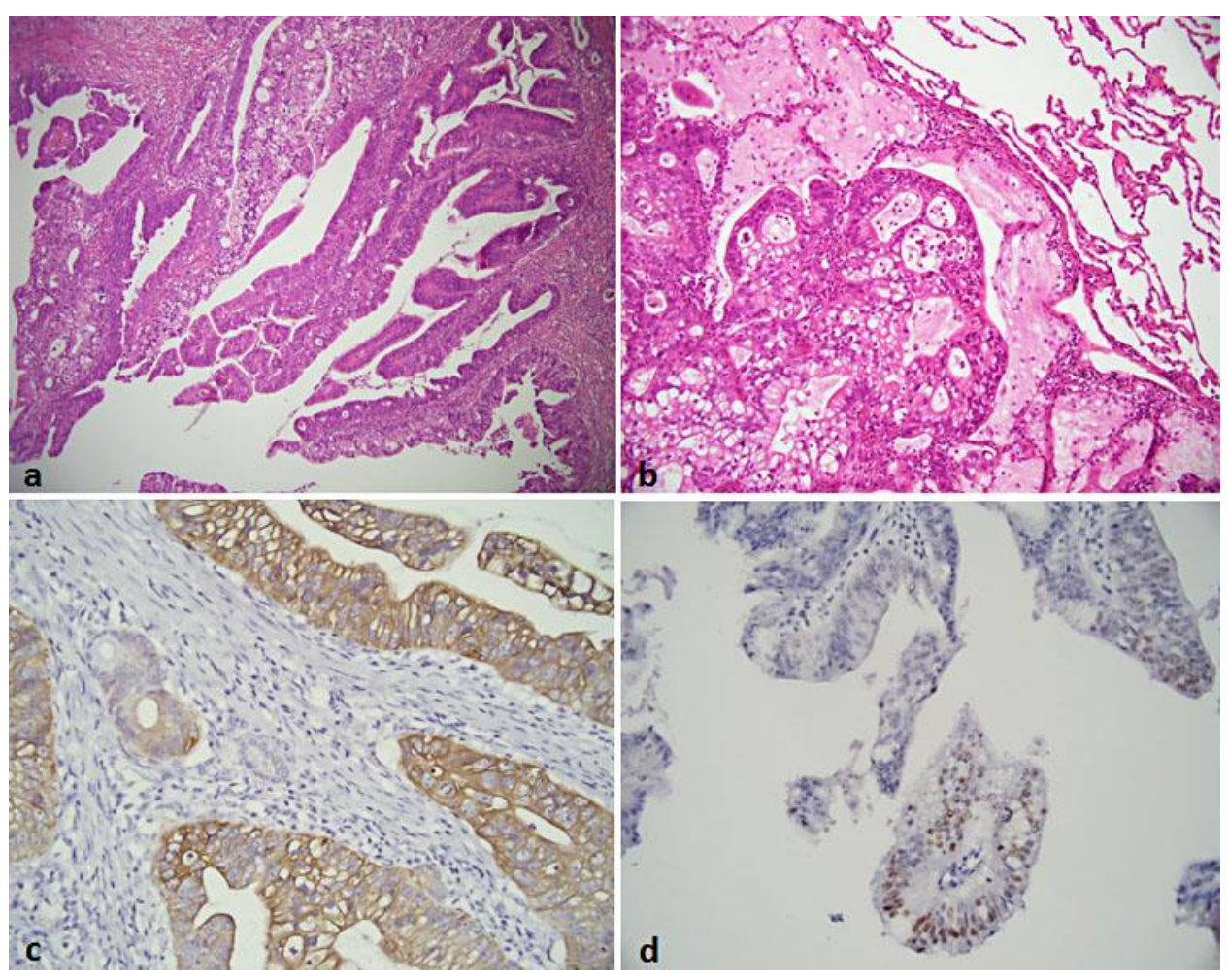

Fig. 1. Histology and immunohistochemistry. a HE staining $(\times 100)$ of the primary tumor sample leading to the diagnosis of an intestinal type. $\mathbf{b}$ HE staining $(\times 200)$ of the resected pulmonary metastasis showing a slightly better morphological differentiation than the primary tumor. c Immunohistochemical staining for CK7 $(\times 400)$ of the primary sample shows a strong positive response of the tumor cells. d Immunohistochemical staining for CDX2 $(\times 400)$ of the primary sample depicts a mostly negative result with very small foci of positive staining. 
Kapp et al.: Exceptional Response to Nanoparticle Albumin-Bound Paclitaxel and Gemcitabine in a Patient with a Refractory Adenocarcinoma of the Ampulla of Vater

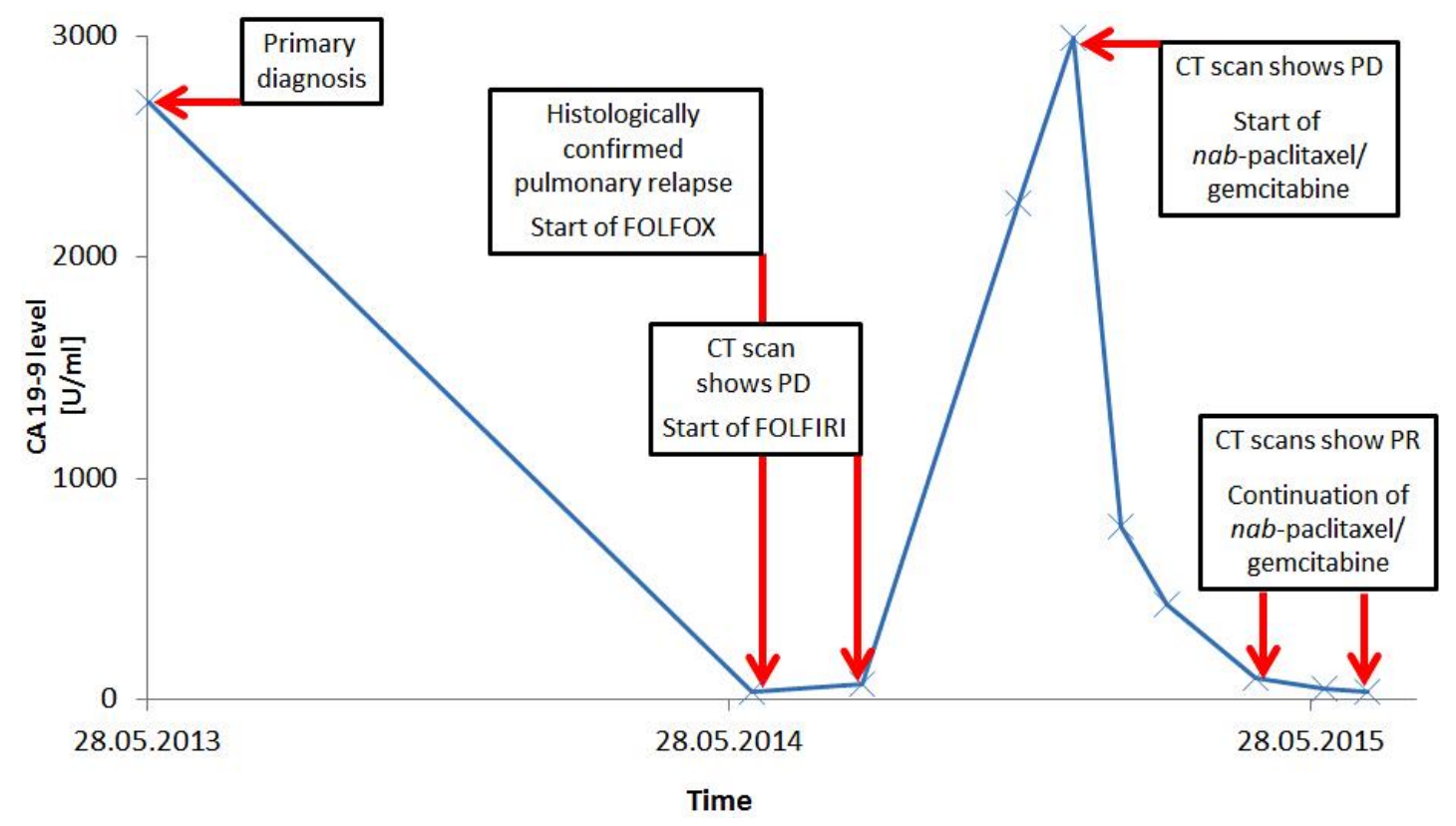

Fig. 2. Course of CA 19-9 level. PD = Progressive disease; $n a b$ - paclitaxel = nanoparticle albumin-bound paclitaxel. 

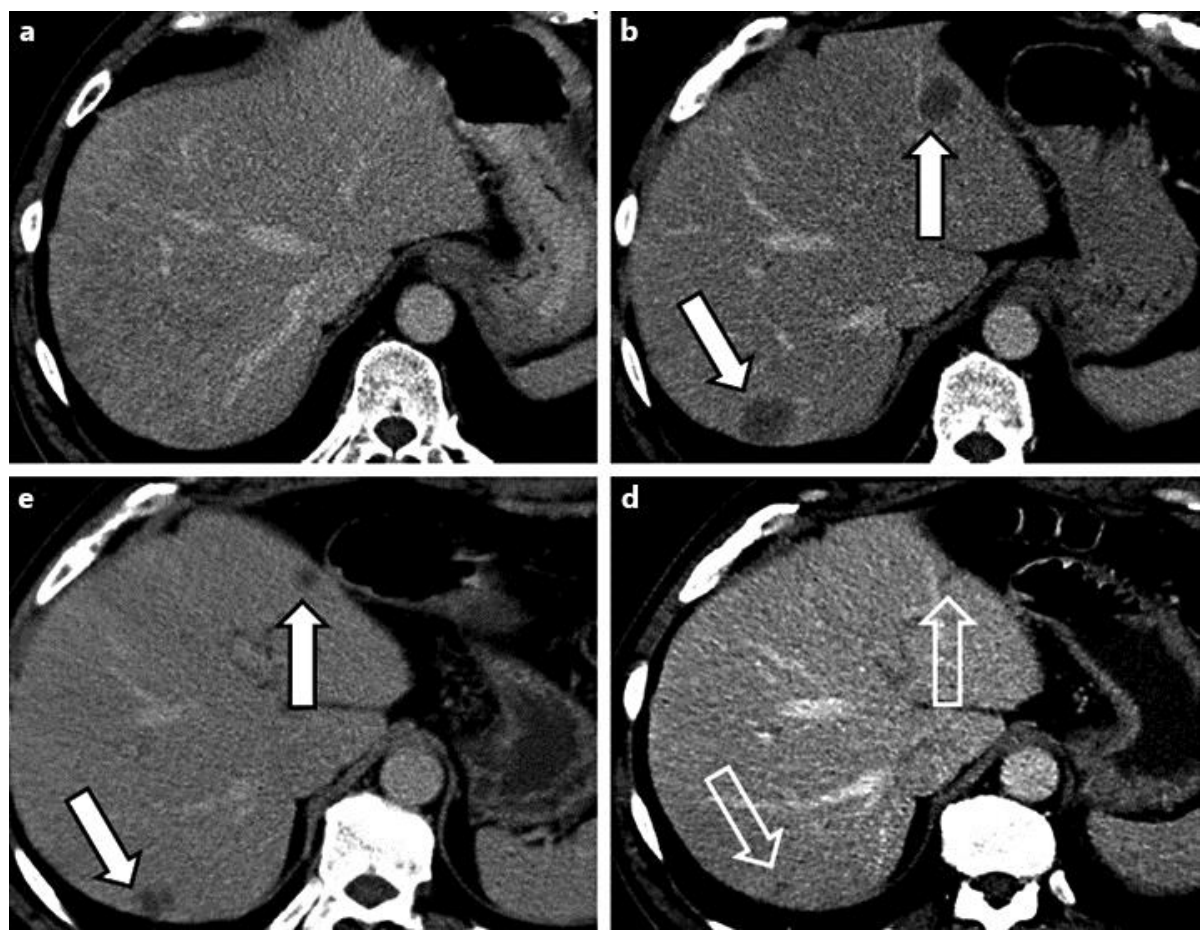

Fig. 3. Initial contrast-enhanced CT of the liver did not display any signs of liver metastases (a). Follow-up CT 15 weeks later readily reveals two liver metastases in segments IVa and VII (bold arrows in b). Therapy with nanoparticle albumin-bound paclitaxel and gemcitabine was initiated. Follow-up CT 13 weeks thereafter demonstrates reduction in size of both liver metastases (c). At further follow-up CT at 17 weeks after initiation of nanoparticle albumin-bound paclitaxel/gemcitabine only residual findings of the former liver metastases are visualized (transparent arrows in $\mathbf{d}$ ). 